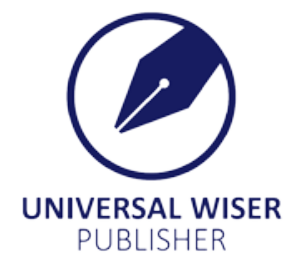

\title{
Correlates of Technology-Assisted Adolescent Dating Violence and Abuse
}

\author{
Karlie E. Stonard \\ University of Wolverhampton \\ E-mail: K.Stonard@wlv.ac.uk
}

\begin{abstract}
Technology-Assisted Adolescent Dating Violence and Abuse (TAADVA) has recently been recognised as new form of violence. However, little is known about the potential risk factors for TAADVA victimisation/perpetration or whether they are similar to those identified for offline Adolescent Dating Violence and Abuse (ADVA). This paper therefore examines the potential correlates of TAADVA victimisation only and perpetration-victimisation (vs. not involved). Findings are reported based on 277 12-18 year old British adolescents who had dated in the last year and completed a series of questionnaires. Findings highlight that correlates associated with ADVA are also related to TAADVA (e.g. past ADVA and having friends with experience of dating violence), however avoidant attachment insecurity was related to male TAADVA, which has not been identified before. Differences were found in some significant correlates for males and females. The findings highlight implications for addressing TAADVA and ADVA through education and awareness about healthy relationships, while considering factors that are associated with TAADVA involvement in prevention and intervention efforts.
\end{abstract}

Keywords: technology-assisted adolescent dating violence, adolescent dating violence

\section{Introduction}

Technology-Assisted Adolescent Dating Violence and Abuse (TAADVA) consists of abusive behaviour perpetrated by an intimate partner that is instigated electronically such as repeated texting or posting sexual pictures of a partner online and may occur between a current or former dating partner ${ }^{[1]}$. Like offline partner violence, TAADVA may include psychological, emotional or sexual abuse as well as coercive and controlling behaviour, the difference being the method through which abusive behaviours are used (e.g. use of a mobile phone or texting, instant messaging, social networking sits, emails, web chats and blogs) ${ }^{[2]}$. Although research on the prevalence of offline Adolescent Dating Violence and Abuse (ADVA) and its correlates/risk factors is well established, particularly in studies conducted in the United States (US), and has been comprehensively reviewed ${ }^{[3-4]}$, the correlates of TAADVA has only relatively recently been explored and is less advanced ${ }^{[5]}$. Research is therefore needed to explore whether factors identified as important in ADVA are also relevant to TAADVA, and whether and how such factors support theoretical explanations of ADVA and TAADVA in order to inform prevention and intervention efforts.

Theoretically, little is known about TAADVA as it is a relatively new phenomenon. Moreover, as a result of unique features of technology (e.g. the accessibility, availability and anonymity), theoretical explanations and risk factors for this type of violence may be different and require unique approaches to prevention and intervention. It has been found for example, that some adolescents only perpetrate dating violence by technology ${ }^{[6]}$. As there is currently no single theory to explain ADVA or TAADVA, more research in needed to build upon and develop knowledge of these issues and subsequent theoretical explanations of both ADVA and TAADVA. This paper therefore seeks to explore whether some factors that have been found to be important in ADVA are also are associated with TAADVA using a sample of British adolescents. Furthermore, it seeks to examine the role of such potential risk factors in terms of their influence on TAADVA experience as a victim only and a perpetrator-victim. This was due to the nature of the sample used in the current study being categorised as being either (1) a victim only, (2) a victim and perpetrator or (3) not involved. Current literature regarding risk factors for ADVA and correlates for TAADVA, along with relevant theoretical perspectives is therefore used to inform the research questions and hypotheses for the current study. 


\subsection{Prevalence of ADVA and TAADVA}

The nature and prevalence of ADVA has been well established and is documented in a comprehensive review of the literature ${ }^{[4]}$. For example, prevalence rates for victimisation in the United States (US) ranged from $6-48 \%$ for physical ADVA, 13-77\% for psychological/emotional ADVA, and 7-13\% for sexual ADVA in the studies reviewed ${ }^{[4]}$. The prevalence in the United Kingdom (UK) were: 9-25\% for female and 12-32\% for male physical victimisation, 32-72\% for female and $27-51 \%$ for male psychological victimisation, and 3-41\% for female and 3-16\% for male sexual victimisation [7-10]

Prevalence rates for TAADVA are also notable, and have been reported to range from 13-64\% for victimisation and $12-70.5 \%$ for perpetration in studies conducted in the US and Europe depending on the type of violence measured and the research design used ${ }^{[11-18]}$. For example, some studies look at TAADVA more broadly while others break this down in terms of individual behaviours. In addition, studies vary in their sample types, ages, the timeframe violence is reported on and whether participants are reporting on their current or recent relationship(s). Similar prevalence rates have been found among samples of young adults. For example. Borrajo et al ${ }^{[19]}$ found that young people (aged 18-30) reported experience both direct aggression (14\% victimisation and $10.6 \%$ perpetration) and controlling behaviour $(75 \%$ victimisation and $82 \%$ perpetration) electronically. In the UK, four studies have acknowledged the role of technology in ADVA. For example, Barter et al ${ }^{[9]}$ asked two questions about TAADVA finding that $12 \%$ of females and $4 \%$ of males reported that a partner had used mobile phones or the Internet to humiliate and threaten them and $42 \%$ of females and $29 \%$ of males reported that partners had frequently checked up on their movements by phone or text. Fox et al ${ }^{[20]}$ asked one question which found that $17 \%$ of adolescents had been checked up on by a partner checking who they have phoned or sent messages to. Barter et al ${ }^{[10]}$ found that $48 \%$ of females and $25 \%$ of males had experienced online emotional TAADVA consisting of six different behaviours. Stonard ${ }^{[21]}$ examined 12 items measuring TAADVA behaviours and found that $73 \%$ of adolescents reported some form of TAADVA victimisation (68\% males and 76\% females) and 50\% reported some form of perpetration (45\% males and 53\% females). Research has also found a high co-occurrence of TAADVA victimisation and perpetration ${ }^{[21-22]}$. These findings indicate that TAADVA is particularly prevalent in adolescent romantic relationships.

Importantly, psychological, controlling, physical and/or sexual ADVA has also been associated with TAADVA [13${ }^{15,21]}$. Additionally, Facebook jealousy has been associated to offline ADVA in terms of being a significant mediator between Facebook use and ADVA ${ }^{[23]}$. Therefore there may be unique factors associated with technology use, in particular, social networking sites, that may result in relationship conflict that may then lead to adolescent involvement in ADVA. This suggests that the two types of violence are related and may have similar risk factors or correlates. However, some adolescents have been found to uniquely experience ADVA or TAADVA. For example, around two-thirds of adolescents reported exclusive TAADVA involvement in Stonard's ${ }^{[21]}$ study, meaning such experiences of dating violence may have unique risk factors or correlates.

\subsection{Attachment theory and its relevance to TAADVA}

Attachment has been used as a theoretical framework to explain the role of risk factors in ADVA and a theoretical framework for adult Intimate Partner Violence (IPV) ${ }^{[24-26]}$. The theory suggests that attachment styles are developed during infancy and characterise individuals, their attitudes and expectations about relationships throughout their life ${ }^{[27-}$

${ }^{28]}$. Attachment styles have been categorised as being secure, anxious-ambivalent, anxious-avoidant, and disorganised ${ }^{[29]}$. Bowlby (1984) theorised that poor experience of supportive relationships in childhood may result in fearful relationships in adulthood characterised by anxious and depressive problems ${ }^{[24]}$. Hazan and Shaver ${ }^{[25]}$ found that more secure lovers described their love experiences as happy and trusting, while avoidant lovers were characterised by a fear of intimacy, and the anxious/ambivalent lover experienced love as involving obsession, extreme sexual attraction and jealousy. In relationships, this fear and emotional reaction (e.g. anxiety or anger) may occur when a relationship is endangered (i.e. risk of loss) and may have a positive function (e.g. to re-establish proximity). From an attachment perspective, when proximity is disrupted, feelings of anxiety, anger or sadness may trigger attachment behaviours designed to re-establish proximity ${ }^{[26]}$. Such feelings may lead to attempts to threaten or coerce a partner psychologically and physically ${ }^{[24]}$.

\subsubsection{Risk factors/correlates that support the attachment theory}

Studies have identified psychological adjustment and personal competency-related factors such as attachment anxiety and high sensitivity to interpersonal rejection (for females) as a risk factor for ADVA perpetration and/or victimisation ${ }^{\text {[30-32] }}$. These findings also support those found in research conducted with older adolescents and adults. For example, Creasey and Hesson-McInnis ${ }^{[33]}$ found that older adolescents $(M=20$ years old) with more insecure and anxious attachment styles were found to have more difficulties regulating emotions when distressed with romantic partners; be more likely to report more anger, sadness, and fear during their interactions with romantic partners; report less confidence in emotional regulation 
during conflicts; and report more difficulties managing conflict. In a study of college students, Follingstad et al ${ }^{[34]}$ identified that while anxious attachment was not directly related to attempts to control one's partner, this relationship was mediated by the person's angry temperament (i.e. anxious attachment was directly related to anger/angry temperament which was related to controlling behaviours). It has also been suggested that problematic combinations of anxiously attached females and avoidant males is associated with male perpetrated IPV in adults ${ }^{[35-36]}$.

Similar findings have also been found in research concerned with TAADVA and violence within adult relationships. Wright ${ }^{[37]}$ explored the links between parental and partner attachment and TAADVA perpetration, finding that maternal avoidance had an indirect effect on TAADVA that was mediated by anxious partner attachment insecurity. Reed et al ${ }^{\text {[38] }}$ found that higher levels of attachment anxiety were associated with more frequent perpetration of TAADVA (defined as electronic intrusion). Furthermore, females reported higher levels of anxiety than males and reported more frequent TAADVA. Reed et al ${ }^{[38]}$ identified that social media may create a 'cycle of anxiety' whereby social media plays a role in triggering relationship anxiety as well as being a tool to monitor a partner online in an attempt to alleviate anxiety . Reed, Tolman, and Safyer ${ }^{[39]}$ similarly found that attachment anxiety was associated with electronic intrusion perpetration in a sample of young adults; in addition to finding that attachment avoidance was negatively associated with electronic intrusion perpetration for women. Qualitative research has also identified the role of anxious emotions and related obsessive and controlling behaviours within the context of young female adolescents' (age 12-13) use of electronic communication technology in romantic relationships ${ }^{[40]}$. Females were also reported to monitor their partner's messages or Facebook accounts, demand passwords to accounts and delete female friends on Facebook due to jealousy or anxiety about a partner's fidelity. Bhogal and Howman ${ }^{[41]}$ also found that attachment anxiety was associated with high levels of TAADVA perpetration. Marshall et al ${ }^{[42]}$ also found that attachment anxiety was positively associated and attachment avoidance negatively associated with Facebook jealousy and surveillance (i.e. checking a romantic partner's Facebook page) in a sample of adults. From the perspective of the attachment theory, anxious attachment insecurity appears to play a role in adolescent experiences of relationships by resulting in abusive, coercive and controlling behaviours in an attempt to maintain those relationships, while attachment avoidance may result in both and increased or reduced likelihood of abusive, monitoring or controlling behaviour as a result of a desire for distancing within a relationship. As the role of attachment appears to be an influential factor in ADVA and TAADVA both theoretically and empirically, this study investigated whether adolescents involved in TAADVA as a victim only or perpetrator-victim reported higher anxious and avoidant attachment insecurity than those not involved and whether this different for males and females.

\subsection{Social learning theory and its relevance to TAADVA}

Social learning theory ${ }^{[43-45]}$ can also be applied to explain several risk factors for ADVA. The social learning theory posits that behaviour is learned and modelled or imitated by observing the behaviour of significant others. Akers ${ }^{[46]}$ suggests the probability that people will engage in or imitate deviant behaviour is increased when they differentially associate with others who commit such behaviour, take on and support accepting attitudes towards the behaviour, and have received or anticipate a relatively greater reward for the behaviour through reinforcement. This reinforcement or reward might be social approval for imitating behaviours that are normalised within ones peer group. The peer context is theorised to provide adolescents with models and expectations about relationships including violence, which then influence adolescents own romantic relationship behaviours ${ }^{[47-48]}$. It has also been suggested that peer influences are most influential during adolescence ${ }^{[49]}$, signifying the potential role of social learning perspective in adolescent experiences of relationships and those that include violence and abuse.

\subsubsection{Risk factors/correlates that support the social learning theory}

Risk factors within the peer and family context and those that are attitudinal in nature appear to be the strongest predictors of ADVA that can support the social learning perspective ${ }^{[45]}$, based on the number of studies to have identified such factors as important ${ }^{[31,50-51]}$. For example, several studies have identified peer influences such as friend dating violence for females ${ }^{[50-52]}$ and for both genders ${ }^{[33-55]}$ as a risk factor for ADVA victimisation and/or perpetration. As it has been suggested that peer influences are most important during adolescence ${ }^{[3,49]}$, particularly in terms of influencing relationship expectations ${ }^{[48]}$, the peer context, including those peer relationships that include dating violence appears to be an important area that is influential in adolescents' own experiences of ADVA. From a social learning perspective, such unhealthy relationship behaviours within the peer context may become normalised as socially learned behaviour and come to be expected within dating relationships.

A recent study into the correlates of TAADVA from a social learning perspective by van Ouytsel, Ponnet, and Walrave ${ }^{[17]}$ found that the perceived social norms of peers, the endorsement of gender stereotypes, and having observed intrusive controlling behaviours by the father are positively related to adolescents' perpetration of digital monitoring 
behaviours. Similarly, norms for violence for boys against girls were a risk factor for TAADVA perpetration in Peskin et al ${ }^{[56]}$ study . However, peer dating violence perpetration was not significant ${ }^{[56]}$. As peer dating violence has been identified as particularly important for ADVA and provides support for the social learning theory, but has not been explored in depth as a correlate for TAADVA and whether the role of this factor is different for males and females, awareness of friend involvement in TAADVA victimisation and perpetration was examined as a correlate for TAADVA victimisation and perpetration-victimisation in the current study.

\subsection{Other risk factors/correlates examined in this study}

Four other factors were identified as important to examine as correlates of TAADVA victimisation/perpetration in the current study due to the empirical importance found in previous research and the potential relevance to the theoretical perspectives examined above: offline ADVA (in order to examine the association between ADVA and TAADVA), perceived relationship closeness, age, and age of dating partner.

\subsubsection{Offline dating violence}

Previous ADVA has been identified as a longitudinal risk factor for future experience of ADVA ${ }^{[57-59]}$. Furthermore, physical, psychological and sexual ADVA have been identified as correlates of TAADVA ${ }^{[11-12,15,18,60]}$. This is important in order to understand whether the risk factors for ADVA are similar to TAADVA or whether TAADVA should be considered as a unique form of violence with unique predictors, in addition to help inform effective prevention strategies and interventions based on such predictors and whether they are important in terms of TAADVA victimisation and/or perpetration. Controlling and physical ADVA was therefore examined in this study in order to confirm if these behaviours were associated with TAADVA victimisation and perpetration-victimisation.

\subsubsection{Relationship closeness}

Adolescents may vary in the frequency and intensity of their romantic relationships ${ }^{[61-62]}$. Females have also been found to express more attachment, care and a higher level of affective intensity in their relationships than males, in addition to reporting relationships that last longer ${ }^{[62]}$. Although, Connolly et al ${ }^{[61]}$ did not find any gender differences in adolescents' dating experience. Reed et al ${ }^{[38]}$ suggest that the relationship context is important to consider when investigating factors that influence TAADVA as it could influence likelihood for problematic relationship behaviours. It is possible that if adolescents have closer or more serious relationships, they may be more likely to experience ADVA or TAADVA within those relationships as a result of spending more time and commitment within those relationships. Another original contribution of this study was therefore to examine whether higher perceived relationship closeness was a correlate of TAADVA victimisation and perpetration-victimisation.

\subsubsection{Age and age of dating partner}

Although an increase in adolescents' own age has been associated with physical and/or emotional ADVA ${ }^{[9]}$, the role of age in TAADVA has been largely inconclusive. Perpetration of TAADVA has been negatively ${ }^{[13]}$ and positively correlated with age for females ${ }^{[38]}$ and both genders ${ }^{[17]}$, as well as being found to be non-significant for TAADVA victimisation, perpetration and mutual TAADVA ${ }^{[22]}$. Therefore, the demographic variable of age was subsequently examined as a correlate for TAADVA victimisation and perpetration-victimisation for males and females in the current study.

Finally, having an older partner (i.e. more than one year older) has been identified as a correlate of physical and emotional ADVA victimisation for females and having a younger partner has been associated with emotional ADVA for males ${ }^{[9]}$. In addition, adolescents who report having older partners have been found to be more likely to experience ADVA (including online emotional abuse) ${ }^{[63]}$. Furthermore, adolescents reporting at least one older partner have been found to be more likely to report online and offline emotional (and sexual for males) dating violence perpetration ${ }^{\text {[63] }}$. Relative age of partner was also a significant predictor of TAADVA perpetration frequency for males in Reed et al.'s ${ }^{\text {[38] }}$ study. However, when Peskin et al ${ }^{[56]}$ examined the age of a dating partner as a risk for TAADVA perpetration it was nonsignificant. Therefore, the age of a dating partner was also examined as a potential correlate of TAADVA victimisation and perpetration-victimisation for males and females in the current study.

\subsection{Summary}

In summary, the current study aimed to extend our understanding of the correlates of TAADVA by examining the role of several factors that have been highlighted as empirically and theoretically important in ADVA and/or TAADVA as reviewed above. As some studies have identified risk factors for victimisation or perpetration only, as well as identifying gender differences in risk factors/correlates, while others have not explored gender, analyses were conducted separately for males and females and for victimisation and perpetration-victimisation separately. The research question addressed in this paper explored whether the following factors: anxious and avoidant attachment; awareness of friend experiences 
of historical and current TAADVA victimisation and perpetration; controlling and physical ADVA victimisation and perpetration; relationship closeness; age; and age of dating partner were: (a) characteristics of those who engage in TAADVA, and (b) independent predictors of TAADVA victimisation or perpetration-victimisation. Two hypotheses were formulated to assess (a) the between groups (TAADVA victimisation, perpetration-victimisation, and none) differences on the 13 predictor variables and (b) examine the independent predictors of TAADVA victimisation and perpetrationvictimisation. Hypothesis and analyses are conducted on TAADVA victims only and TAADVA perpetrator-victims vs. none due to low number of adolescents reporting experiences of TAADVA perpetration only.

\subsection{Hypotheses}

Specifically, it was expected that:

Hypothesis 1: There will be significant between groups (TAADVA victims only, perpetrator-victims, and none) differences on the 13 predictor variables such that those involved in TAADVA as a victim and/or perpetrator-victim will also report being insecurely attached (avoidant and anxious), having friends with experience of TAADVA (historical and current) victimisation and perpetration, having experience of controlling and physical ADVA victimisation and perpetration, higher perceived relationship closeness, being older, and having older dating partners.

Hypothesis 2: Attachment insecurity (avoidant and anxious), having friends with experience of TAADVA (historical and current) victimisation and perpetration, experience of controlling and physical ADVA victimisation and perpetration, higher perceived relationship closeness, being older, and having older dating partners will independently predict group membership as a TAADVA victim only or a perpetrator-victim vs. none.

\section{Method}

\subsection{Participants}

A total 469 British adolescents (52\% female; $88 \%$ White British Ethnicity) aged between 12-18 years ( $\mathrm{M}=13.9$ years; Median $=14$ years; $\mathrm{SD}=1.27$ ) were recruited using opportunity and purposive sampling through schools, youth clubs and via snowballing in Central England. Data were collected in 2013-2014. Findings reported in this paper are limited to those adolescents $(59 \% ; \mathrm{n}=277)$ with past year dating relationship experience and who provided data for all of the variables used in the subsequent analysis. Respondents reported on their experiences with a current or past boyfriend or a girlfriend within the last 12 months.

\subsection{Procedure}

Ethical clearance was granted from Coventry University's Research Ethics Committee. All secondary schools and youth clubs within the local area of the researcher and reasonable travel distance were contacted by phone and/or email. Gatekeeper consent was gained from three secondary schools and seven youth clubs. This included year 8-9 classes in the schools and a range of ages (12-18 years) within the youth clubs. Parents/guardians were informed about the research, its aims and the focus of the questionnaires by letter and consent for their child's participation was sought using an optout procedure. Recruitment of adolescents was generally very high in schools and only three adolescents were opted out by their parents and one adolescent did not consent to take part across two of the schools. Adolescent consent rates within the youth clubs were lower than that in the schools, likely due to the more informal environment (exact figures were not recorded). Participants completed a series of pencil-and-paper questionnaires administered in the same way in each setting using the same introductions, instructions, delivered by the same person, with the same process.

\subsection{Measures}

\subsubsection{TAADVA}

The TAADVA victimisation and perpetration surveys each consisted of 12 abusive, threatening, monitoring or controlling TAADVA behaviours (e.g. insults; embarrassing or humiliating a partner; sharing a partner's personal information or pictures; pressure to engage in unwanted sexting; and checking messages, contact histories or friend lists) that could be experienced via a range of electronic communication technologies (e.g. call, text, instant message, social networking site, picture message, video chat, email chatroom, website/blog) developed from a review of literature and an earlier pilot study ( Stonard et al. ${ }^{[4]}$ and Stonard et al. ${ }^{[40]}$ ). The full list of TAADVA behaviours asked about in the questionnaire is provided in the Appendix. Response options ranged in frequency from 'never', 'at least once', 'monthly', 'fortnightly', 'weekly', 'daily', to 'hourly' and the timeframe was the last 12 months. The data from the TAADVA victimisation questions were grouped together and recoded as categorical (e.g. no experience $=0$ and yes experience $=1$ ), as was done for the perpetration data. 


\subsubsection{Controlling ADVA}

The Controlling Behaviors Scale ${ }^{[64]}$ was used to measure past year controlling ADVA and consisted of 12 items for each victimisation and perpetration using four of the five original subscales which were aggregated (threats, intimidation, emotional abuse and isolation). Questions included for example, making threats to harm or leave a partner, humiliating or putting a partner down, and restricting the amount of time a partner spends with friends or family. Response options ranged from 'never', 'hardly ever', 'sometimes', 'often', to 'very often'. The data from the controlling ADVA victimisation questions were grouped together and recoded as categorical (e.g. no experience $=0$ and yes experience $=1$ ), as was done for the perpetration data.

\subsubsection{Physical ADVA}

Fifteen items from each of the Victimisation and Perpetration in Dating Relationships Scales (VDRS/PDRS) ${ }^{[65]}$ were used to measure past year physical ADVA (e.g. slapping, kicking, choking, punching and more serious behaviours such as assaulting with a weapon). Response options ranged from 'never', '1-3 times', '4-9 times', to 'ten or more times'. The data from the physical ADVA victimisation questions were grouped together and recoded as categorical (e.g. no experience $=0$ and yes experience $=1$ ), as was done for the perpetration data.

\subsubsection{Friend experience of TAADVA}

A set of eight questions was devised to measure awareness of the number of friends with experience of historical and current TAADVA (two questions for each historical and current TAADVA victimisation and perpetration). These two questions represented non-sexual and sexual TAADVA behaviours. Response options ranged from ' 0 friends', ' 1 friend', ' 2 friends', ' 3 friends', to 'more than 3 friends'. The friend TAADVA variables were combined into four variables based on whether this was awareness of friend experience of historical or current TAADVA victimisation or perpetration and responses were recoded as categorical (e.g. no awareness of friend experience $=0$ and awareness of friend experience $=1$ ).

\subsubsection{Attachment}

The 36-item Experiences in Close Relationships-Relationship Structures questionnaire ${ }^{[66]}$ was used to measure global scores for anxious and avoidant attachment (including to a mother, father, friend and partner). Participants are asked to rate their responses on a seven point scale from 'strongly disagree' to 'strongly agree' to questions such as whether they discuss their problems and concerns with each person, whether they find it easy to depend on each person, and whether they fear these people may abandon them or not care for them. The first six items for each person make up the avoidant attachment scale whereby higher scores suggest that respondents are more securely attached. The last three items make up the anxious attachment scale where higher scores suggest that respondents are more insecurely attached. The Cronbach's alpha score was $\alpha=.87$ for the avoidant attachment scale and $\alpha=.91$ for the anxious attachment scale for this study.

\subsubsection{Relationship closeness}

The final scale used was the 12-item Unidimensional Relationship Closeness Scale ${ }^{[67]}$ regarding adolescents perceived relationship closeness (e.g. whether they think their relationship was close, whether they tell important personal things to their girlfriend or boyfriend, and whether they have a strong connection with their girlfriend or boyfriend) measured on a seven point scale from 'strongly disagree' to 'strongly agree'. This 12-item measure's Cronbach's alpha score for this study was $\alpha=.96$.

\subsubsection{Other demographic questions}

Respondents were also asked how old their current or most recent girlfriend/boyfriend was and to state their age within the demographic information section.

\subsection{Analytical strategy}

Data screening revealed that the data breached assumptions of linearity, homogeneity and/or Box's test of normality. Descriptive frequencies of the 13 predictor variables for the categories of TAADVA group membership for males and females are summarised for contextual information. In order to answer the first hypothesis, the impact of group membership on the 13 predictor variables was examined using Kruskal-Wallis ANOVA or Chi-Square/Fisher's Exact (FE) Tests depending on the type of predictor variable (e.g. continuous or categorical). Post hoc tests were then performed (e.g. Mann-Whitney $U$ tests for the continuous variables) on significant findings. To test the second hypothesis, multinomial logistic regression was conducted to examine if any of these significant correlates of TAADVA independently predicted TAADVA group membership.

\section{Results}

The prevalence of TAADVA for males was as follows: victim only $(25 \%)$, perpetrator only $(2 \%)$, perpetrator-victim 
(43\%), and not involved (31\%). For females this was: victim only (22\%), perpetrator only ( $0 \%)$, perpetrator-victim (53\%), and not involved (24\%). Analysis of the 13 predictor variables in relation to TAADVA is reported separately for males and females and for TAADVA victim only and perpetrator-victim roles vs. none as a result of the small sample reporting TAADVA perpetration only.

\subsection{Between groups comparisons of the 13 predictor variables}

\subsubsection{Males}

The findings for the 13 predictor variables for each TAADVA group for males are summarised in Table 1a. As seen in Table 1a, there was a significant effect of TAADVA by group (non-involved, victim, and perpetrator-victim) on six of the 13 predictor variables (avoidant attachment, age, having friends with experience of current TAADVA victimisation, selfreported controlling ADVA victimisation and perpetration, and self-reported physical ADVA perpetration).

Mann-Whitney U tests were used to follow up the significant findings from the Kruskal-Wallis analyses of continuous variables (avoidant attachment and age) in order to determine where these differences were. A Bonferroni correction was applied meaning all effects are reported at a .03 level of significance. Avoidant attachment scores and the age of those not involved in TAADVA did not significantly differ from TAADVA victims only (Table 1b). This suggests that male TAADVA victims and non-involved adolescents did not significantly differ in their avoidant attachment and age.

Avoidant attachment scores of those not involved in TAADVA did significantly differ from TAADVA perpetratorvictims (Table 1c). The age of those not involved in TAADVA did not significantly differ from TAADVA perpetratorvictims (Table 1c). This suggests that males with no experience of TAADVA were more securely attached on the avoidant scale than TAADVA perpetrator-victims who were more insecure. Moreover, TAADVA perpetrator-victims did not significantly differ from those not involved in TAADVA in terms of age.

Post hoc test were conducted to follow up the significant Chi-square and FE tests of the significant categorical variables (having friends with experience of current TAADVA victimisation, self-reported controlling ADVA victimisation and perpetration and physical ADVA perpetration) to determine where these significant differences were. Post hoc analyses revealed non-significant differences between groups for the friend current TAADVA victimisation and selfreported controlling ADVA perpetration variables. Post hoc analyses did however reveal significant between groups differences for the controlling ADVA victimisation variable, with males in the TAADVA perpetrator-victim category being overrepresented in the controlling ADVA victimisation experience category $(\mathrm{z}=2.53, \mathrm{p}=.01)$; and the physical ADVA perpetration variable, with males in the TAADVA perpetrator-victim category being overrepresented in the physical ADVA perpetration experience category $(\mathrm{z}=2.06, \mathrm{p}=.04)$. There was therefore significant association between TAADVA perpetration-victimisation vs. none for three of the 13 predictor variables for males: avoidant attachment, controlling ADVA victimisation, and physical ADVA perpetration.

\subsubsection{Females}

The findings for the 13 predictor variables for each TAADVA group for females are summarised in Table 2a. As seen in Table 2a, there was a significant effect of TAADVA by group (non-involved, victim, and perpetrator-victim) on nine of the 13 predictor variables (relationship closeness, age, having friends with experience of historical and current TAADVA victimisation and historical TAADVA perpetration, self-reported controlling ADVA victimisation and perpetration and physical ADVA victimisation and perpetration).

Mann-Whitney U tests were used to follow up the significant findings from the Kruskal-Wallis analyses of continuous variables (relationship closeness and age) in order to determine where these differences were. A Bonferroni correction was applied meaning all effects are reported at a .03 level of significance. Perceived relationship closeness scores and the age of those not involved in TAADVA did not significantly differ from TAADVA victims only (Table $2 b$ ). This suggests that female TAADVA victims and non-involved adolescents did not significantly differ in their perceived relationship closeness and age.

Perceived relationship closeness scores of those not involved in TAADVA did not significantly differ from TAADVA perpetrator-victims (Table 2c). The age of those not involved in TAADVA did significantly differ from TAADVA perpetrator-victim (Table 2c). This suggests that females with experience of TAADVA as a perpetrator-victim were older than those not involved in TAADVA. Moreover, TAADVA perpetrator-victims did not significantly differ from those not involved in TAADVA in terms their perceived relationship closeness.

Post hoc test were conducted to follow up the significant Chi-square and FE tests of the significant categorical variables (having friends with experience of historical and current TAADVA victimisation, friend experience of historical TAADVA perpetration, self-reported controlling ADVA victimisation and perpetration and physical ADVA victimisation and perpetration) to determine where these significant differences were. Post hoc analyses revealed non-significant 
differences between groups for the friend historical TAADVA victimisation variable, with only those females in the TAADVA perpetrator-victim category approaching significance $(\mathrm{z}=-1.92, \mathrm{p}=.05)$. If this were significant, this would suggest that female TAADVA perpetrator-victims were also more likely to report having friends with experience of historical TAADVA victimisation rather than not reporting this factor. Post hoc analyses did however reveal significant between groups differences for the other six variables. For example, females in the TAADVA victim only category were significantly underrepresented in the friends with current TAADVA victimisation experience category $(\mathrm{z}=-2.19, \mathrm{p}=$ .03 ) and those in the TAADVA perpetrator-victim category were significantly overrepresented in the friends with current TAADVA victimisation experience category $(\mathrm{z}=2.32, \mathrm{p}=.02)$. Females in the TAADVA victim only category were also significantly underrepresented in the friends with historical TAADVA perpetration experience category $(\mathrm{z}=-2.45, \mathrm{p}=.01)$ and those in the TAADVA perpetrator-victim category were significantly overrepresented in the friends with historical TAADVA perpetration experience category $(\mathrm{z}=2.40, \mathrm{p}=.02)$.

Females in the TAADVA not involved category were significantly underrepresented in the controlling ADVA victimisation experience category $(\mathrm{z}=-3.46, \mathrm{p}=.001)$ and those in the TAADVA perpetrator-victim category were significantly overrepresented in the controlling ADVA victimisation experience category $(z=3.49, \mathrm{p}=.001)$. Females in the TAADVA not involved category were also significantly underrepresented in the controlling ADVA perpetration experience category $(\mathrm{z}=-2.19, \mathrm{p}=.03)$ and those in the TAADVA perpetrator-victim category were significantly overrepresented in the controlling ADVA perpetration experience category $(\mathrm{z}=2.76, \mathrm{p}=.01)$. Females in the TAADVA not involved and TAADVA victim only categories were significantly underrepresented in the physical ADVA victimisation experience category $(\mathrm{z}=-2.10, \mathrm{p}=.04$ and $\mathrm{z}=-2.11, \mathrm{p}=.04$ respectively $)$ and those in the TAADVA perpetratorvictim category were significantly overrepresented in the physical ADVA victimisation experience category $(\mathrm{z}=2.80$, $\mathrm{p}=.01$ ). Finally, females in the TAADVA victim only category were significantly underrepresented in the physical ADVA perpetration experience category $(\mathrm{z}=-2.40, \mathrm{p}=.02)$ and those in the TAADVA perpetrator-victim category were significantly overrepresented in the physical ADVA perpetration experience category $(\mathrm{z}=2.00, \mathrm{p}=.04)$. Therefore, an increase in these factors appears to be related to TAADVA perpetration-victimisation only. There were therefore significant associations between the TAADVA groups for a total of seven of the 13 predictor variables for females: age, friend current TAADVA victimisation, friend historical TAADVA perpetration, self-reported controlling ADVA victimisation and perpetration, and physical ADVA victimisation and perpetration.

\subsection{Independent predictors of TAADVA victimisation and perpetration-victimisation}

This section of analyses examined which of the previously identified significant correlates (three for males and seven for females) of TAADVA victimisation and perpetration-victimisation were the strongest independent predicators. The reference group was no experience of TAADVA.

\subsubsection{Males}

A multinomial logistic regression analysis was conducted in order to explore which of the previously significant predictor variables independently predicted TAADVA victimisation and perpetration-victimisation for male adolescents (Table 3). A test of the final model fit for males with the three factors was significant $(\chi 2(6)=28.407, p<.001)$, suggesting that the model explains a significant amount of the original variability. Having experience of physical ADVA perpetration independently significantly predicted whether male adolescents were a TAADVA victim only (OR $=5.82 \mathrm{E}-08, \mathrm{p}<.001)$. Avoidant attachment and controlling ADVA victimisation did not significantly predict TAADVA victimisation only (Table 3). Avoidant attachment insecurity did however independently significantly predict whether adolescents were a TAADVA perpetrator-victim $(\mathrm{OR}=0.51, \mathrm{p}<.05)$, as did the controlling ADVA victimisation variable $(\mathrm{OR}=0.22, \mathrm{p}<.05)$. Physical ADVA perpetration did not independently predict TAADVA perpetration-victimisation (Table 3 ).

\subsubsection{Females}

A multinomial logistic regression analysis was conducted in order to explore which of the previously identified variables of interest independently predicted TAADVA group membership (victim and perpetrator-victim) for females (Table 4). A test of the final model fit with the seven factors was significant $(\chi 2(14)=87.539, p<.001)$, suggesting the model explains a significant amount of the original variability. Only the controlling ADVA victimisation variable independently significantly predicted whether female adolescents were a TAADVA victim only $(\mathrm{OR}=0.05, \mathrm{p}<.001)$. The other six factors were non-significant: age; friend current TAADVA victimisation; friend historical TAADVA perpetration; controlling ADVA perpetration; physical ADVA victimisation; and physical ADVA perpetration (Table 4). Three of the seven factors independently significantly predicted whether female adolescents were a TAADVA perpetrator-victim: controlling ADVA victimisation ( $\mathrm{OR}=0.03, \mathrm{p}<.001)$; physical ADVA victimisation $(\mathrm{OR}=0.10, \mathrm{p}<.05)$; and physical ADVA perpetration $(\mathrm{OR}=13.36, \mathrm{p}<.05)$. The other four variables were non-significant: age; friend current TAADVA 
victimisation; friend historical TAADVA perpetration; and controlling ADVA perpetration (Table 4).

\subsubsection{Post-hoc power analysis}

A post-hoc power analysis was conducted based on the alpha size (0.05), effect size (.5), and sample size of the multinomial logistic regression models. This medium to large effect size is recommended by Cohen ${ }^{\left[{ }^{[8]}\right]}$. Post-hoc power analysis revealed that the achieved power for the significant independent predictors for males were as follows: physical ADVA perpetration (100\%) for the TAADVA victim only category, and avoidant attachment $(95 \%)$ and controlling ADVA victimisation $(100 \%)$ for the TAADVA perpetrator-victim category, well within the suggested acceptable level of power of at least $80 \%{ }^{[69]}$. The post-hoc power analysis for the non-significant predictors ranged from $44 \%-82 \%$. For females, the achieved power for the significant independent predictors were: controlling ADVA victimisation (100\%) for the TAADVA victim only category, and controlling ADVA victimisation (100\%), physical ADVA victimisation (100\%) and physical ADVA perpetration $(100 \%)$ for the TAADVA perpetrator-victim category. The post-hoc power analysis for the nonsignificant predictors ranged from $0 \%-100 \%$.

\section{Discussion}

This paper aimed explore whether risk factors identified as being related to ADVA were also correlates of TAADVA victimisation and perpetration-victimisation, and whether these factors independently predicted TAADVA victimisation and perpetration-victimisation. It was theorised that risk factors that support the attachment (such as avoidant and anxious attachment) and social learning theoretical perspectives (such as awareness of friend experiences of dating violence), that have been identified as important in explaining ADVA perpetration/victimisation would also be related to adolescent experience of TAADVA perpetration/victimisation. These factors were chosen to examine due to their potential theoretical importance, potential for influencing prevention and intervention, and due to there being few studies regarding these potential influences in terms of TAADVA and type of TAADVA involvement for males and females. In addition, other variables thought to be risk factors for ADVA such as experiencing past ADVA, perceived relationship closeness, age, and age of dating partner were examined to see if they were related to TAADVA due to a gap in literature exploring these factors based on TAADVA victimisation and perpetration-victimisation for males and females, particularly these latter three factors.

The first hypothesis tested whether there was a significant between groups (TAADVA victims only, perpetratorvictims, and none) difference on the 13 predictor variables examined and expected that those involved in TAADVA will also report more attachment insecurity (avoidant and anxious), having friends with experience of TAADVA (historical and current) victimisation and perpetration, having experience of controlling and physical ADVA victimisation and perpetration, higher perceived relationship closeness, being older, and having older dating partners. This was partially supported. Male TAADVA perpetrator-victims were more likely than those not involved to report more avoidant attachment insecurity in addition to self-reported experiences of controlling ADVA victimisation and physical ADVA perpetration. Female TAADVA perpetrator-victims were more likely than those not involved to report awareness of friend experiences of current TAADVA victimisation and historical TAADVA perpetration, self-reported experience of controlling and physical ADVA victimisation and perpetration, and were older in age. Females who were TAADVA victims only were less likely than those not involved to report having friends with current experience of TAADVA victimisation and historical experience of TAADVA perpetration, and self-reported experience of physical ADVA perpetration.

The finding that avoidant attachment insecurity was a correlate for male TAADVA perpetration-victimisationis in contrast to previous research with young adults/adults that found attachment avoidance was negatively associated with TAADVA ${ }^{[42,39]}$. However avoidant attachment has been related to adult/young adult IPV victimisation ${ }^{[70]}$ and perpetration ${ }^{[71]}$. It is interesting that anxious attachment was not significant, considering research has found this factor to be associated with ADVA ${ }^{[30,32]}$ and TAADVA ${ }^{[41,38,37]}$. Drawing on the attachment theory perspective, it may be that avoidant male adolescents use TAADVA to distance themselves from a partner or become victims due a partner's wish to pursue closeness within the relationship, a partner that may be more anxiously attached, as has been found in research with adults in terms of adult IPV ${ }^{[72,36]}$. More research is needed on the role of anxious and avoidant attachment in TAADVA, particularly attachment avoidance as limited research has explored this with adolescents making this finding in the current study a novel contribution to understanding the role of attachment in TAADVA for males.

The finding that friend dating violence was a correlate for female TAADVA supports previous research on the role of friend ADVA as a risk factor for ADVA ${ }^{[50-52]}$. However, this is in contrast to research that found this factor to be important for both males and females ${ }^{[53-55]}$. Thus, peer TAADVA may be an important factor in shaping female adolescents' expectations about relationships including TAADVA. This lends support to the social learning perspective 
as having friends with experience of TAADVA may influence female adolescents' own expectations and normalisations of what relationships should be like. The use of violence may be observed, learned and reinforced within the peer group meaning such relationship behaviours are considered acceptable. It is possible that female adolescents may discuss their relationships more than boys or that friends have a greater influence as role models however further research would be needed to explore this. Another possibility is that having been involved in TAADVA may mean that female adolescents are more likely to discuss such experiences with friends whom may have then disclosed their experiences of TAADVA. Future longitudinal research could help to clarify this influence.

These findings highlight that controlling and physical ADVA victimisation and/or perpetration and TAADVA was experienced in combination by adolescents with a dual role of TAADVA experience (i.e. perpetrator-victim) within the last 12 months. This confirms that found in previous studies ${ }^{[11-12,18]}$. However, this is the first study to explore adolescents' experience of these factors in terms of the experience of victimisation and perpetration-victimisation combined. These findings confirm that TAADVA and ADVA are connected, but causal relationships cannot be concluded.

The finding that being older was associated with TAADVA for females also supports previous research with regards to ADVA ${ }^{[9]}$ and TAADVA ${ }^{[17,38]}$. It is interesting that the age difference of adolescents' dating partner variable was not significant, contrary to Barter et al.'s ${ }^{[63]}$ recent study that found that having an older dating partner was associated with ADVA including online emotional abuse. Nevertheless, these findings highlight that some predictors found to be associated with ADVA are also relevant to TAADVA, in particular, as a perpetrator-victim.

The second hypothesis tested whether those variables that were previously significant in the between groups comparisons independently predicted group membership as a TAADVA victim only or a perpetrator-victim vs. none, and was also partially supported. Having experience of physical ADVA perpetration significantly and independently predicted being a TAADVA victim only for males in the multinomial logistic regression analysis. This may suggest that males who have been abusive physically to a partner then become victims of TAADVA in retaliation, or perhaps are abusive physically in response to TAADVA victimisation. Furthermore, avoidant attachment insecurity and controlling ADVA victimisation significantly predicted male TAADVA perpetration-victimisation Although casual relationships cannot be confirmed, this highlights the association between TAADVA and ADVA experiences. These findings also suggest that male adolescents who score more securely on the avoidant attachment measure may be more open and less distant in their relationships, meaning there is less need to monitor or control a partner or to be monitored or controlled themselves. On the other hand, more avoidant adolescents may use violence to distance oneself from intimacy or as a result of poor relationship functioning ${ }^{[24-26]}$. Being more avoidant in relationships may suggest an avoidance of relationships altogether, or this could mean that (potentially more preoccupied) partners of avoidant males pursue information, for example, by monitoring messages to learn information about their partner or in an attempt to get closer resulting in TAADVA ${ }^{[73]}$. This would reflect that found in research with adults by Doumas et al ${ }^{[36]}$ whereby the combination of avoidant males with anxiously attached female partners was associated with male perpetration of IPV. Again, it is surprising that only avoidant attachment was significant and for males only, given the findings from previous research where it was found that females expressed awareness of or personal experiences of anxious, obsessive and controlling feelings or behaviours within the context of romantic relationships ${ }^{[40]}$. Similarly, research with adults has found a relationship between anxious attachment and IPV victimisation and/or perpetration ${ }^{[70,74-76]}$; however, this was not this case in this study with adolescents.

For females, experience of controlling ADVA victimisation significantly independently predicted TAADVA as a victim only, suggesting an overlap between controlling ADVA victimisation and TAADVA victimisation. This

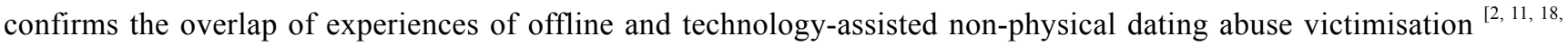

${ }^{60]}$. Controlling victimisation, and physical ADVA victimisation and perpetration also significantly and independently predicted experience of TAADVA as a perpetrator-victim, signifying that not only is there an overlap between ADVA and TAADVA victimisation, but this was also related to TAADVA perpetration. It is possible that TAADVA perpetration may have been carried out in response or retaliation to offline ADVA victimisation, however longitudinal research is needed to confirm this. In addition, it appears that TAADVA perpetration-victimisation is experienced alongside controlling ADVA victimisation and physical ADVA victimisation and perpetration as a wider repertoire of abuse. These findings can help inform typologies of TAADVA and ADVA in order to develop future theoretical explanations and preventative strategies.

\subsection{Implications}

The findings from this paper highlight several important theoretical implications that can inform future research. First, the finding that avoidant attachment insecurity independently predicted TAADVA as a perpetrator-victim for males is noteworthy and requires further examination. For example, it is not known whether this relationship between avoidant attachment and TAADVA is a result of male adolescents' avoidant attachments with parents, partners and 
friends collectively as a result of the global anxious attachment measure, or whether certain aspects of this variable are more strongly related to self-reported TAADVA. Further exploration of this factor could help to inform both theoretical explanations of TAADVA and prevention efforts that address unhealthy attachments and relationship behaviours. It would be useful to examine the role attachment in TAADVA and ADVA in more detail including any notable gender differences. Although significant gender differences in the prevalence of attachment styles and its association with adult IPV has not been found ${ }^{[25,76]}$, the role of attachment anxiety and avoidance in TAADVA and ADVA may be different for males and females based on the findings of this study. As anxious attachment was not statistically significant like it has been found in previous research regarding ADVA ${ }^{[30,32]}$ and TAADVA ${ }^{[37,41]}$, it would be useful to explore other psychological adjustment and personal competency-related factors rather than attachment directly such as jealousy or sensitivity to interpersonal rejection ${ }^{[31]}$. Future research should consider such personal and relational factors that may be linked with attachment and their potential direct and indirect associations with TAADVA, particularly considering recent findings regarding the role of social media-related jealousy and TAADVA ${ }^{[23]}$. Other interesting avenues of research could build on the findings of Fox and Warber ${ }^{[7]}$ that attachment style predicted relational uncertainty and electronic surveillance, and the findings of Marshall et al ${ }^{[42]}$ that anxiety was positively associated, and avoidance negatively associated with Facebook jealousy and surveillance. Additionally, it would be interesting to explore whether the effect of attachment varies in terms of its influence on TAADVA and ADVA. The initial significant finding regarding relationship closeness for females may also provide a promising line of further inquiry as a main effect or as a moderator or mediator with other factors such as attachment.

The finding that friend TAADVA was associated with female TAADVA perpetration-victimisation raises important theoretical insights into the influence of peers, the role of social learning and the potential development of attitudes that tolerate TAADVA as normal behaviour within adolescent romantic relationships. This finding can help to inform future theoretical explanations of TAADVA and identifies the peer context as a potentially important avenue for raising awareness of unhealthy relationships. The finding also highlights the potential role of peers as attachment figures and role models in the development of relationship expectations. Further research that explores the role of situational factors such as peer influences in addition to attachments to friends, partners and parents as a potential mediating factor may also prove an interesting line of enquiry to assess the significance of these attachments, peer role models, and personal relationship functioning.

Finally, the finding that controlling and physical ADVA was related to self-reported TAADVA highlights and confirms the overlap between ADVA and TAADVA and a need to consider online and offline forms of violence when attempting to explain and manage violence in adolescent romantic relationships. As there is a connection between ADVA and TAADVA and their correlates, this needs to be recognised in future prevention and intervention efforts. It is likely that for many adolescents, TAADVA is an extension of ADVA as a result of the availability of more tools to abuse and control a partner. Adolescents' may perpetrate or experience ADVA initially and then become a victim or perpetrator of TAADVA as technology allows a partner to abuse when they are apart as well as when together in person. Technology might be used as an additional method of abuse, control or coercion. Likewise, a partner may be abusive or controlling via technology and then progress to also being abusive or becoming a victim in person. Further longitudinal research could explore which type of abuse is experience/used first and explore how this then progresses to other types of abuse. Experience of both ADVA and TAADVA may represent a more harmful impact if abusive and controlling behaviour is experienced in both contexts. Subsequently, when one type of abuse is identified as being present, practitioners should look for signs of the other.

Despite this overlap, the nature and impact of TAADVA that is experienced exclusively may need to be treated uniquely in policy and practice. For example, the role of avoidant attachment has not been identified as a risk factor for ADVA like anxious attachment has. However avoidant attachment has been related to adult offline partner violence ${ }^{[70-}$ ${ }^{71]}$. Therefore, TAADVA may represent a preferred method to abuse or control a partner from a distance for those with avoidant representations of attachments. Future research should continue to expand our understanding on the similarities and differences between each type of dating violence in order to develop our awareness, theoretical explanations and subsequent appropriate policy and practice responses.

Several implications for policy and practice are raised from the findings in this paper. These findings highlight a need for prevention and intervention efforts to address attachment avoidance (for males). In addition, education regarding healthy relationships, ADVA/TAADVA awareness, managing conflict and relationship functioning would prove useful. Education that addresses such issues in the peer context may be effective in changing attitudes towards ADVA/TAADVA and encouraging healthy relationships, help-seeking and bystander intervention. There has been evidence to suggest that bystander initiatives show promise for changing social norms towards domestic and sexual violence and prevention at universities particularity in the US ${ }^{[78]}$, such approaches may also prove useful in school settings to help challenge 
attitudes accepting of ADVA and TAADVA and to encourage disclosures, reporting and help-seeking. School settings and other organisations and cyberspaces that young people attend and interact with each other are an ideal place to integrate interventions. Practitioners should also be mindful of the different roles of involvement (i.e. victim and/or perpetrator) adolescents may have in TAADVA and the influencing factors involved with its nature and management. Furthermore, prevention and intervention efforts should take into account that factors associated with TAADVA may differ for males and females. For example, friend TAADVA was particularly important for females while avoidant attachment was associated with TAADVA for males.

It is important to consider both offline ADVA and TAADVA in future prevention and intervention efforts in order to address all forms of violence that adolescents may experience and be affected by. Although UK government policy has recently acknowledged 16-17 year olds in its definition of domestic violence ${ }^{[79]}$, this does not specifically detail the role of technology in TAADVA, or the evidence that young people under the age of 16 may be a victim and/or perpetrator of TAADVA or ADVA. The UK government has demonstrated its commitment to tackling violence against women and girls at a national and international level ${ }^{[80-81]}$, yet more needs to be done to address this not only in terms of defining this in policy but also addressing it through compulsory education and raising awareness publically to challenge cultural and social acceptance of ADVA and TAADVA. The current study provides a deeper understanding of the relatively unexplored issue of TAADVA and its correlates that can be used to help inform such strategies.

\subsection{Limitations and future directions}

As with any research the findings should be considered within the context of its limitations, however, these limitations highlight opportunities for future research. Data collected with self-report surveys is subject to response bias. For example, participants may reply in a socially desirable direction or not wish to disclose personal information about TAADVA or ADVA victimisation/perpetration. Simon et al ${ }^{[82]}$ suggest that gender differences in prevalence reports could be due to sex differences in the willingness of adolescents to report ADVA, which is likely to be influenced by the perceived social acceptability of violence. As such, the authors argue that females may be less willing to disclose victimisation and males less willing to disclose perpetration due to the notion that male violence is less socially acceptable. Another limitation is that it is not known whether respondents were reporting on a current or past dating relationship or multiple relationships within the last 12 months. Furthermore, the study is cross-sectional meaning causal relationships cannot be determined. Additionally, as data collection for the study was conducted during 2013-14, technology use by adolescents may have changed (e.g. smartphone ownership, the apps used by adolescents etc.), which may affect the experience of TAADVA. Although the power analyses suggested the sample size was adequate, the sample was fairly small and future research should try to replicate the results using a larger sample. Finally, demographic questions were not asked regarding the adolescents sexual orientation or whether they had any disability, which future research should consider. Despite this, this paper offers unique contributions to our understanding of correlates of TAADVA and whether factors thought to be associated with offline ADVA can also be applied to TAADVA. Future research can draw on these findings, limitations and implications to advance our knowledge of TAADVA and its risk factors further.

\section{References}

[1] Centers for Disease Control and Prevention. Understanding Teen Dating Violence; 2014. Available from: https://www. cdc.gov/violenceprevention/pdf/teen-dating-violence-2014-a.pdf [Accessed 9th May 2020].

[2] Cutbush S, Williams J, Miller S et al. Electronic Dating Aggression Among Middle School Students: Demographic Correlates and Associations with Other Types of Violence. Poster presented at the American Public Health Association annual meeting, October 27-31; 2012. San Francisco, CA, viewed April 7, 2015. Available from: http://www.rti.org/ pubs/apha12_cutbush_poster.pdf. [Accessed 9th May 2020].

[3] Leen E, Sorbring E, Mawer M et al. Prevalence, Dynamic Risk Factors and the Efficacy of Primary Interventions for Adolescent Dating Violence: An International Review. Aggression and Violent Behavior. 2013; 18: 159-174.

[4] Stonard K E, Bowen E, Lawrence T R et al. The Relevance of Technology to the Nature, Prevalence and Impact of Adolescent Dating Violence and Abuse: A Research Synthesis. Aggression and Violent Behavior. 2014; 19: $390-417$.

[5] Stonard K E. Explaining Adolescent Dating Violence and Abuse (ADVA) and Technology-Assisted Adolescent Dating Violence and Abuse (TAADVA): Risk Factors and Correlates. Advancements in Educational Psychology. 2019; 1(1): 23-66.

[6] Korchmaros J D, Ybarra M L, Langhinrichsen-Rohling J et al. Perpetration of Teen Dating Violence in a Networked Society. Cyberpsychology, Behavior, and Social Networking. 2013; 16: 561-567.

[7] Hird M J. An Empirical Study of Adolescent Dating Aggression in the U.K. Journal of Adolescence. 2002; 23(1): 69- 
78.

[8] Burman M, Cartmel F. Young Peoples Attitudes Towards Gendered Violence. NHS Health Scotland: Edinburgh; 2005. Available from: http://www.healthscotland.com/uploads/documents/475-GenderedViolence.pdf [Accessed 9th May 2020].

[9] Barter C, McCarry M, Berridge D et al. Partner Exploitation and Violence in Teenage Intimate Relationships. London: NSPCC; 2009.

[10] Barter C, Wood M, Aghtaie N et al. Briefing Paper 2: Incidence Rates and Impact of Experiencing Interpersonal Violence and Abuse in Young People's Relationships. Safeguarding Teenage Intimate Relationships: Connecting Online and Offline Contexts and Risks. Funded by DAPHNE III European Commission; 2015a. Available from: https://www.safenet.bg/images/sampledata/files/STIR-Briefing-Paper-2-English-final.pdf [Accessed 9th May 2020].

[11] Cutbush S, Ashley O S, Kan M L et al. Electronic Aggression Among Adolescent Dating Partners: Demographic Correlates and Associations with Other Types of Violence. Poster presented at the American Public Health Association annual meeting, November 6-10. Denver, CO; 2010. Available from http://www.rti.org/pubs/apha10_cutbush_poster. pdf [Accessed 9th May 2020].

[12] Dick R N, McCauley H L, Jones K A et al. Cyber Dating Abuse Among Teens Using School-Based Health Centers. Pediatrics. 2014; 134: 1560-1567.

[13] Doucette H, Collibee C, Hood E et al. Perpetration of Electronic Intrusiveness Among Adolescent Females: Associations With In-Person Dating Violence. Journal of Interpersonal Violence. 2018. Available from: doi: $10.1177 / 0886260518815725$.

[14] Morelli M, Bianchi D, Chirumbolo A et al. The cyber dating violence inventory. Validation of a new scale for online perpetration and victimization among dating partners. European Journal of Developmental Psychology. 2017. Available from: doi: 10.1080/17405629.2017.1305885.

[15] Temple J R, Choi H J, Brem M et al. The Temporal Association between Traditional and Cyber Dating Abuse among Adolescents. Journal of Youth and Adolescence. 2016; 45: 340-349.

[16] van Ouytsel J, Torres E, Choi H J et al. The Associations Between Substance Use, Sexual Behaviors, Bullying, Deviant Behaviors, Health, and Cyber Dating Abuse Perpetration. The Journal of School Nursing. 2017; 33: 116-122. Available from: doi:10.1177/1059840516683229.

[17] van Ouytsel J, Ponnet K, Walrave M. Cyber Dating Abuse: Investigating Digital Monitoring Behaviors Among Adolescents From a Social Learning Perspective. Journal of Interpersonal Violence. 2017; 1-22. Available from: doi:10.1177/0886260517719538.

[18] Zweig J M, Dank M, Yahner J et al. The Rate of Cyber Dating Abuse Among Teens and How it Relates to Other Forms of Teen Dating Violence. Journal of Youth and Adolescence. 2013; 42: 1063-1077.

[19] Borrajo E, Gámez-Guadix M, Pereda N et al. The development and validation of the cyber dating abuse questionnaire among young couples. Computers in Human Behavior. 2015; 48: 358-365.

[20] Fox C L, Corr M L, Gadd D et al. Young Teenagers' Experiences of Domestic Abuse. Journal of Youth Studies. 2014; 17(4): 510-526.

[21] Stonard K E. The Prevalence and Overlap of Technology-Assisted and Offline Adolescent Dating Violence. Current Psychology. 2018.

[22] Smith K, Cénata J M, Lapierrea A et al. Cyber dating violence: Prevalence and correlates among high school students from small urban areas in Quebec. Journal of affective disorders. 2018; 234: 220-223.

[23] Daspe M-È, Vaillancourt-Morel M-P, Lussier Y et al. Facebook Use, Facebook Jealousy, and Intimate Partner Violence Perpetration. Cyberpsychology, Behavior, and Social Networking. 2018; 21(9): 549-555.

[24] Bowlby J. Violence in the Family as a Disorder of the Attachment and Caregiving Systems. The American Journal of Psychoanalysis. 1984; 4: 9-27.

[25] Hazan C, Shaver P. Romantic Love Conceptualised as an Attachment Process. Journal of Personality and Social Psychology. 1987; 52(3): 511-524.

[26] Hazan C, Shaver P R. Attachment as an Organisational Framework for Research on Close Relationships. Psychological Inquiry. 1994; 5(1): 1-22.

[27] Bowlby J. Attachment and Loss: Vol. 1. Attachment. New York: Basic Books; 1969.

[28] Bowlby, J. The Making and Breaking of Affectional Bonds. London: Tavistock Publications; 1979.

[29] Ainsworth M D S, Blehar M, Waters E et al. Patterns of Attachment: A Psychological Study of the Strange Situation. Hillsdale, NJ: Erlbaum; 1978.

[30] Miga E M, Hare A, Allen J P et al. The Relation of Insecure Attachment States of Mind and Romantic Attachment Styles to Adolescent Aggression in Romantic Relationships. Attachment and Human Development. 2010; 12: 463-sdx zrzswzdxegykm"pjngvtfasdxcsegtcvdrwq481.

[31] Moretti M M, Bartolo T, Craig S et al. Gender and the Transmission of Risk: A Prospective Study of Adolescent Girls 
Exposed to Maternal Versus Paternal Interparental Violence. Journal of Research on Adolescence. 2014; $24:$ 80-92.

[32] Ulloa E C, Martinez-Arango N, Hokoda A. Attachment Anxiety, Depressive Symptoms, and Adolescent Dating Violence Perpetration: A Longitudinal Mediation Analysis. Journal of Aggression, Maltreatment \& Trauma. 2014; 23: 652-669.

[33] Creasey G, Hesson-McInnis M. Affective Responses, Cognitive Appraisals, and Conflict Tactics in Late Adolescent Romantic Relationships: Associations With Attachment Orientations. Journal of Counseling Psychology. 2001; 48(1): 85-96.

[34] Follingstad D R, Bradley R G, Helff C M et al. A Model for Predicting Dating Violence: Anxious Attachment, Angry Temperament, and Need for Relationship Control. Violence and Victims. 2002; 17(1): 35-47.

[35] Godbout N, Dutton D G, Lussier Y A et al. Early Exposure to Violence, Domestic Violence, Attachment Representations, and Marital Adjustment. Personal Relationships. 2009; 16: 365-384.

[36] Doumas D M, Pearson C L, Elgin J E et al. Adult Attachment as a Risk Factor for Intimate Partner Violence: The "'Mispairing" of Partners' Attachment Styles. Journal of Interpersonal Violence. 2008; 23: 616-634.

[37] Wright M F. Cyber Aggression Within Adolescents' Romantic Relationships: Linkages to Parental and Partner Attachment. Journal of Youth Adolescence. 2014; 44: 37-47.

[38] Reed L A, Tolman R M, Ward L M et al Keeping tabs: Attachment anxiety and electronic intrusion in high school dating relationships. Computers in Human Behavior. 2016; 58: 259-268.

[39] Reed L A, Tolman R M, Safyer P. Too close for comfort: Attachment insecurity and electronic intrusion in college students' dating relationships. Computers in Human Behavior. 2015; 50: 431-438.

[40] Stonard K, Bowen E, Walker K et al. "They'll Always Find a Way to Get to You": Technology Use in Adolescent Romantic Relationships and Its Role in Dating Violence and Abuse. Journal of Interpersonal Violence. 2017; 32: 2083-2117.

[41] Bhogal M S, Howman J H. Mate Value Discrepancy and Attachment Anxiety Predict the Perpetration of Digital Dating Abuse. Evolutionary Psychological Science. 2018; 5(1): 113-120.

[42] Marshall T C, Bejanyan K, Di Castro G et al. Attachment styles as predictors of Facebook-related jealousy and surveillance in romantic relationships. Personal Relationships. 2013; 20(1); 1-22.

[43] Bandura A. Social Learning Theory. New York: General Learning Press; 1971.

[44] Bandura A. Aggression: A Social Learning Analysis. Oxford: Prentice-Hall; 1973.

[45] Bandura A. Social Learning Theory. Englewood Cliffs, NJ: Prentice-Hall; 1977.

[46] Akers R L. Social Learning and Social Structure: A General Theory of Crime and Deviance. Boston: Northeastern University Press; 1998.

[47] Connolly J, Goldberg A. Romantic Relationships in Adolescence: The Role of Friends and Peers in their Emergence and Development. In: Furman W, Brown B B, Feiring. C. (eds). The Development of Romantic Relationships in Adolescence. Cambridge: Cambridge University Press; 1999. p. 266-290.

[48] Furman W. The Emerging Field of Adolescent Romantic Relationships. Current Directions in Psychological Science. 2002; 11(5): 177-180.

[49] Harris J R. Where Is the Child's Environment? A Group Socialization Theory of Development. Psychological Review. 1995; 102(3): 458-489.

[50] Arriaga X B, Foshee V A. Adolescent Dating Violence: Do Adolescents Follow in Their Friends', or Their Parents', Footsteps?. Journal of Interpersonal Violence. 2004; 19: 162-184.

[51] Foshee V A, Linder G F, MacDougall J E et al. Gender Differences in the Longitudinal Predictors of Adolescent Dating Violence. Preventive Medicine. 2001; 32: 128-141.

[52] Foshee V A, Benefield T S, Ennett S T et al. Longitudinal Predictors of Serious Physical and Sexual Dating Violence Victimization During Adolescence. Preventive Medicine. 2004; 39(5): 1007-1016.

[53] Ellis W E, Chung-Hall J, Dumas T M. The Role of Peer Group Aggression in Predicting Adolescent Dating Violence and Relationship Quality. Journal of Youth Adolescence. 2013; 42: 487-499.

[54] Foshee V A, McNaughton-Reyes H L, Ennett S T. Examination of Sex and Race Differences in Longitudinal Predictors of the Initiation of Adolescent Dating Violence Perpetration. Journal of Aggression, Maltreatment \& Trauma. 2010; 19: 492-516.

[55] Foshee V A, Benefield T S, McNaughton-Reyes H L et al. The Peer Context and the Development of the Perpetration of Adolescent Dating Violence. Journal of Youth Adolescence. 2013; 42: 471-486.

[56] Peskin M F, Markham C M, Shegog R et al. Prevalence and Correlates of the Perpetration of Cyber Dating Abuse among Early Adolescents. Journal of Youth and Adolescence. 2017; 46(2): 358-375.

[57] McNaughton-Reyes H L, Foshee V A, Bauer D J et al. Proximal and Time-Varying Effects of Cigarette, Alcohol, Marijuana and Other Hard Drug Use on Adolescent Dating Aggression. Journal of Adolescence. 2014; 37(3): 281289. 
[58] Temple J R, Shorey R C, Fite P et al. Substance Use as a Longitudinal Predictor of the Perpetration of Teen Dating Violence. Journal of Youth Adolescence. 2013; 42: 596-606.

[59] Tschann J M, Pasch L A, Flores E et al. Nonviolent Aspects of Interparental Conflict and Dating Violence Among Adolescents. Journal of Family Issues. 2009; 30: 295-319.

[60] Zweig J M, Lachman P, Yahner J et al. Correlates of Cyber Dating Abuse Among Teens. Journal of Youth Adolescence. 2014; 43(8): 1306-1321.

[61] Connolly J, Craig W, Goldberg A et al. Mixed-Gender Groups, Dating, and Romantic Relationships in Early Adolescence. Journal of Research on Adolescence. 2004; 14: 185-207.

[62] Shulman S, Scharf M. Adolescent Romantic Behaviors and Perceptions: Age-and Gender-Related Differences, and Links with Family and Peer Relationships. Journal of Research on Adolescence. 2000; 10: 99-118.

[63] Barter C, Wood M, Aghtaie N et al. Briefing Paper 3: Risk and Protective (Predictive) Factors for Interpersonal Violence and Abuse Victimisation and Instigation. Safeguarding Teenage Intimate Relationships: Connecting Online and Offline Contexts and Risks. Funded by DAPHNE III European Commission; 2015b. Available from: https://www. safenet.bg/images/sampledata/files/STIR-Briefing-Paper-3-English-Final-.pdf [Accessed 9th May 2020].

[64] Graham-Kevan N, Archer J. Physical Aggression and Control in Heterosexual Relationships: The Effect of Sampling. Violence and Victims. 2003; 18: 181-196.

[65] Foshee V A, Linder G F, Bauman K E et al. The Safe Dates Project: Theoretical Basis, Evaluation Design, and Selected Baseline Findings. American Journal of Preventative Medicine. 1996; 12: 39-47.

[66] Fraley R C, Heffernan M E, Vicary A M et al. The Experiences in Close Relationships-Relationship Structures Questionnaire: A Method for Assessing Attachment Orientations Across Relationships. Psychological Assessment. 2011; 23: 615-62.

[67] Dibble J L, Levine T R, Park H S. The Unidimensional Relationship Closeness Scale (URCS): Reliability and Validity Evidence for a New Measure of Relationship Closeness. Psychological Assessment. 2012; 24: 565-572.

[68] Cohen J. Quantitative Methods in Psychology: A Power Primer. Psychological Bulletin. 1992; 112(1): 115-159.

[69] Cohen J. Statistical Power Analysis for the Behavior Sciences. 2nd ed. Hillsdale, NJ: Erlbaum; 1988.

[70] Bonache H, Gonzalez-Mendez R, Krahé B. Adult Attachment Styles, Destructive Conflict Resolution, and the Experience of Intimate Partner Violence. Journal of interpersonal violence. 2016. Available from: doi: $10.1177 / 0886260516640776$.

[71] Yarkovsky N. Experiences of Dating Violence in Emerging Adult Couples: The Role of Attachment Style and Emotion Regulation. Electronic Theses and Dissertations. University of Windsor; 2016. Available from: https://pdfs. semanticscholar.org/fbb7/151e07dc8844c6f68291a1e0ffbf85bef70c.pdf [Accessed 9th May 2020].

[72] Allison C K, Bartholomew K, Mayseless O et al. Love as a Battlefield: Attachment and Relationship Dynamics in Couples Identified for Male Partner Violence. Journal of Family Issues. 2008; 29(1): 125-150.

[73] Baker C K, Carreño P K. Understanding the Role of Technology in Adolescent Dating and Dating Violence. Journal of Child and Family Studies. 2016; 25: 308-320.

[74] Bond S B, Bond M. Attachment Styles and Violence Within Couples. Journal of Nervous and Mental Disease. 2004; 192(12): 857-863.

[75] Fournier B, Brassard A, Shaver P R. Adult Attachment and Male Aggression in Couple Relationships: The DemandWithdraw Communication Pattern and Relationship Satisfaction as Mediators. Journal of Interpersonal Violence. 2010; 26(10): 1982-2003.

[76] Henderson A J Z, Bartholomew K, Trinke S J et al. When Loving Means Hurting: An Exploration of Attachment and Intimate Abuse In a Community Sample. Journal of Family Violence. 2005; 20(4): 219-230.

[77] Fox J, Warber K M. Social networking sites in romantic relationships: attachment, uncertainty, and partner surveillance on Facebook. Cyberpsychology, Behavior, and Social Networking. 2014; 17(1): 3-7.

[78] Fenton R A, Mott H L, McCartan K et al. A Review of Evidence for Bystander Intervention to Prevent Sexual and Domestic Violence in Universities. London: Public Health England; 2016.

[79] Home Office. Cross-Government Definition of Domestic Violence: A Consultation Summary of Responses. London: Home Office; 2012.

[80] Home Office. A Call to End Violence Against Women and Girls: Action Plan 2014. London: Home Office; 2014.

[81] Home Office. Ratification of the Council of Europe Convention on Combating Violence Against Women and Domestic Violence (Istanbul Convention) Report on Progress. London: Home Office; 2017.

[82] Simon T R, Miller S, Gorman-Smith D et al. Physical Dating Violence Norms and Behavior Among Sixth-Grade Students from four U.S. Sites. Journal of Early Adolescence. 2010; 30(3): 395-409.

\section{Appendix}

TAADVA questionnaire behaviour items (repeated for victimisation and perpetration) 
1. Insulting, mean or hurtful personal comments e.g. called you names, put downs etc.

2. Comments or acts that were intended to embarrass, humiliate or shame you e.g. spreading rumours

3. Shared or distributed private or personal information/images/videos etc.

4. Threatening comments or behaviours that were intended to threaten harm, intimidate or bully you

5. Been contacted to check on you and ask you where you are, what you are doing and who you are with.

6. Asked or pressured you to engage in sexual acts or to send messages/pictures that you did not want.

7. Been sent sexual or inappropriate messages/pictures etc. that you did not want

8. Checked your messages, contact histories or friend lists/networks

9. Demanded your passwords to check messages, contact histories or friend lists/networks

10. Deleted or removed contacts or friends or ex-partners

11. Made you feel afraid not to respond to a call, text, message etc.

12. Prevented you from using electronic communication technology or from talking to others

\section{Tables}

Table 1a. Between group comparisons on 12 predictor variables for males $(n=106-112)$

\begin{tabular}{|c|c|c|c|c|}
\hline & $\begin{array}{c}\text { None } \\
M(S D) / \% \text { (n) }\end{array}$ & $\begin{array}{l}\text { TAADVA Victim Only } \\
\qquad(S D) / \%(\mathbf{n})\end{array}$ & $\begin{array}{c}\text { TAADVA } \\
\text { Perp-Vict } \\
M(S D) / \%(n)\end{array}$ & Kruskal-Wallis / $\mathbf{X}^{2}$ or FE \\
\hline Anxious attachment & $2.46(1.19)$ & $2.09(1.19)$ & $2.49(1.33)$ & $\mathrm{H}(2,106)=5.55, p=.28$ \\
\hline Avoidant attachment & $4.97(0.94)$ & $4.50(0.87)$ & $4.33(1.01)^{* *}$ & $\mathrm{H}(2,106)=8.18, p=.02 *$ \\
\hline Relationship closeness & $4.43(1.37)$ & $4.39(1.62)$ & $5.02(1.32)$ & $\mathrm{H}(2,112)=4.77, p=.09$ \\
\hline Age & $13.89(1.39)$ & $13.61(0.74)$ & $14.63(1.75)$ & $\mathrm{H}(2,112)=8.55, p=.01 * *$ \\
\hline $\begin{array}{c}\text { Friend historical TAADVA } \\
\text { victim }\end{array}$ & $37(13)$ & $46(13)$ & $47(23)$ & $\begin{array}{c}\mathrm{X}^{2}(2,112)=0.91, p=.64, \text { Cramer's } \mathrm{V} \\
=.09\end{array}$ \\
\hline Friend current TAADVA victim & $17(6)$ & $0(0)$ & $16(8)$ & $\begin{array}{c}\mathrm{FE}(112)=6.28, p=.04, \text { Cramer's } V \\
=.22^{*}\end{array}$ \\
\hline $\begin{array}{l}\text { Friend historical TAADVA } \\
\text { perpetrator }\end{array}$ & $17(6)$ & $7(2)$ & $18(9)$ & $\begin{array}{c}\mathrm{X}^{2}(2,112)=1.90, p=.39, \text { Cramer's } \\
V=.13\end{array}$ \\
\hline $\begin{array}{l}\text { Friend current TAADVA } \\
\text { perpetrator }\end{array}$ & $11(4)$ & $4(1)$ & $14(7)$ & $\begin{array}{c}\mathrm{FE}(112)=2.07, p=.37, \text { Cramer's } V \\
=.14\end{array}$ \\
\hline Controlling ADVA victim & $12(4)$ & $7(2)$ & $42(20)^{* *}$ & $\begin{array}{l}\mathrm{X}^{2}(2,109)=15.19, p=.001 \\
\text { Cramer's } V=.37 * * *\end{array}$ \\
\hline Controlling ADVA perpetrator & $18(6)$ & $21(6)$ & $44(21)$ & $\begin{array}{c}\mathrm{X}^{2}(2,109)=7.45, p=.02, \text { Cramer's } V \\
=.26^{*}\end{array}$ \\
\hline Physical ADVA victim & $12(4)$ & $29(8)$ & $31(15)$ & $\begin{array}{c}\mathrm{X}^{2}(2,109)=4.13, p=.13, \text { Cramer's } V \\
=.20\end{array}$ \\
\hline Physical ADVA perpetrator & $0(0)$ & $4(1)$ & $17(8)^{*}$ & $\begin{array}{c}\mathrm{FE}(108)=7.66, p=.01, \text { Cramer's } V \\
=.28^{* *}\end{array}$ \\
\hline Age of dating partner (older) & $3(1)$ & $11(3)$ & $12.5(6)$ & $\begin{array}{c}\mathrm{FE}(110)=2.35, p=.33, \text { Cramer's } V \\
=.15\end{array}$ \\
\hline
\end{tabular}

Note: *Significant at the .05 level; **.01; ***.001 (2-tailed)

Table 1b. Post hoc Mann-Whitney comparisons between TAADVA victims and non-involved males

\begin{tabular}{|c|c|c|c|c|c|c|c|c|}
\hline & None $M d n$ & $\begin{array}{c}\text { Non } \\
\text { Range }\end{array}$ & Victim Mdn & VictimRange & $\begin{array}{c}\text { Victims } \\
\text { Mann-Whitney } \\
U \\
\end{array}$ & $z$-score & $p$-value & $\begin{array}{l}\text { Effect } \\
\operatorname{size}(r)\end{array}$ \\
\hline Avoidant attachment & 5.00 & $3.25-6.96$ & 4.54 & $3.00-6.42$ & 322.50 & -1.828 & .068 & -0.24 \\
\hline Age & 14.00 & $12.00-18.00$ & 13.50 & $13.00-16.00$ & 458.50 & -0.473 & .636 & -0.06 \\
\hline
\end{tabular}

Note: *Significant at the .03 level (2-tailed) Bonferroni corrected

Table 1c. Post hoc Mann-Whitney comparisons between TAADVA perpetrator-victims and non-involved males

\begin{tabular}{|c|c|c|c|c|c|c|c|c|}
\hline & \multirow[b]{2}{*}{ None Mdn } & \multirow[b]{2}{*}{ NoneRange } & \multicolumn{6}{|c|}{ Perpetrator-Victims } \\
\hline & & & $\begin{array}{c}\text { Perp-Vic } \\
M d n\end{array}$ & $\begin{array}{c}\text { Perp-Vic } \\
\text { Range }\end{array}$ & $\begin{array}{c}\text { Mann-Whitney } \\
U\end{array}$ & $z$-score & $p$-value & $\underset{(r)}{\text { Effect size }}$ \\
\hline Avoidant attachment & 5.00 & $3.25-6.96$ & 4.19 & $2.67-6.67$ & 483.00 & -2.744 & $.006^{*}$ & -0.31 \\
\hline Age & 14.00 & $12.00-18.00$ & 14.00 & $12.00-18.00$ & 634.50 & -2.123 & .034 & -0.23 \\
\hline
\end{tabular}

Note: *Significant at the .03 level (2-tailed) Bonferroni corrected 
Table 2a. Between group comparisons on the 13 predictor variables for females $(n=147-156)$

\begin{tabular}{|c|c|c|c|c|}
\hline & $\begin{array}{c}\text { None } \\
M(S D) / \% \text { (n) }\end{array}$ & $\begin{array}{c}\text { TAADVA Victim Only } \\
M(S D) / \% \text { (n) } \\
\end{array}$ & $\begin{array}{l}\text { TAADVA Perp-Vict } \\
M(S D) / \%(n)\end{array}$ & Kruskal-Wallis / $\mathbf{X}^{2}$ \\
\hline Anxious attachment & $2.16(0.98)$ & $2.30(1.34)$ & $2.69(1.34)$ & $\mathrm{H}(2,147)=4.13, p=.13$ \\
\hline Avoidant attachment & $5.07(1.05)$ & $4.70(0.85)$ & $4.55(0.91)$ & $\mathrm{H}(2,147)=5.74, p=.06$ \\
\hline Relationship closeness & $4.41(1.14)$ & $4.22(1.22)$ & $4.79(1.46)$ & $\mathrm{H}(2,152)=7.34, p=.03 *$ \\
\hline Age & $13.82(1.27)$ & $13.66(1.08)$ & $14.53(1.36)^{* *}$ & $\mathrm{H}(2,156)=15.90, p=.000 * * *$ \\
\hline Friend historical TAADVA victim & $34(13)$ & $43(15)$ & $69(57)$ & $\begin{array}{c}\mathrm{X}^{2}(2,156)=14.95, p=.001 \\
\text { Cramer's } V=.31 * * *\end{array}$ \\
\hline Friend current TAADVA victim & $13(5)$ & $6(2)^{*}$ & $36(30)^{*}$ & $\begin{array}{c}\mathrm{X}^{2}(2,156)=, p=.000, \text { Cramer's } V \\
=.32 * * *\end{array}$ \\
\hline $\begin{array}{l}\text { Friend historical TAADVA } \\
\text { perpetrator }\end{array}$ & $13(5)$ & $3(1)^{* *}$ & $35(29)^{*}$ & $\begin{array}{l}\mathrm{X}^{2}(2,156)=17.05, p=.000 \\
\quad \text { Cramer's } V=.33^{* * *}\end{array}$ \\
\hline $\begin{array}{l}\text { Friend current TAADVA } \\
\text { perpetrator }\end{array}$ & $5(2)$ & $3(1)$ & $14.5(12)$ & $\begin{array}{c}\mathrm{FE}(156)=4.33, p=.11, \text { Cramer's } V \\
=.18\end{array}$ \\
\hline Controlling ADVA victim & $8(3)^{* * *}$ & $25(8)$ & $72.5(58) * * *$ & $\begin{array}{l}\mathrm{X}^{2}(2,150)=50.51, p=.000 \\
\text { Cramer's } V=.58^{* * *}\end{array}$ \\
\hline Controlling ADVA perpetrator & $26(10)^{*}$ & $27(9)$ & $74(60)^{* *}$ & $\begin{array}{c}\mathrm{X}^{2}(2,152)=33.94, p=.000, \text { Cramer's } \\
V=.47 * * *\end{array}$ \\
\hline Physical ADVA victim & $8(3)^{*}$ & $6(2)^{*}$ & $40.5(32)^{* *}$ & $\begin{array}{c}\mathrm{X}^{2}(2,149)=22.16, p=.000, \text { Cramer's } \\
V=.39 * * *\end{array}$ \\
\hline Physical ADVA perpetrator & $13(5)$ & $0(0)^{*}$ & $27.5(22)^{*}$ & $\begin{array}{c}\mathrm{X}^{2}(2,150)=12.52, p=.002, \text { Cramer's } \\
V=.29^{* *}\end{array}$ \\
\hline Age of dating partner (older) & $19(7)$ & $29(10)$ & $32.5(27)$ & $\begin{array}{c}\chi^{2}(2,154)=2.11, p=.35, \text { Cramer's } V \\
=.12\end{array}$ \\
\hline
\end{tabular}

Note: *Significant at the .05 level; **.01; ***.001 (2-tailed)

Table 2b. Post hoc Mann-Whitney comparisons between TAADVA victims and non-involved females

\begin{tabular}{|c|c|c|c|c|c|c|c|c|}
\hline & \multirow[b]{2}{*}{ NoneMdn } & \multirow[b]{2}{*}{ None Range } & \multirow[b]{2}{*}{ Victim Mdn } & \multicolumn{2}{|c|}{ Victims } & \multirow[b]{2}{*}{$z$-score } & \multirow[b]{2}{*}{$p$-value } & \multirow[b]{2}{*}{$\begin{array}{c}\text { Effect size } \\
(r)\end{array}$} \\
\hline & & & & VictimRange & $\begin{array}{c}\text { Mann-Whitney } \\
U\end{array}$ & & & \\
\hline $\begin{array}{l}\text { Relationship } \\
\text { closeness }\end{array}$ & 4.08 & $1.58-6.25$ & 4.42 & $1.25-6.25$ & 577.50 & -0.412 & .681 & -0.05 \\
\hline Age & 14.00 & $12.00-18.00$ & 13.00 & $12.00-18.00$ & 614.00 & -0.606 & .545 & -0.07 \\
\hline
\end{tabular}

Note: *Significant at the .03 level (2-tailed) Bonferroni corrected

Table 2c. Post hoc Mann-Whitney comparisons between TAADVA perpetrator-victims and non-involved females

\begin{tabular}{|c|c|c|c|c|c|c|c|c|}
\hline & \multirow[b]{2}{*}{ None $M d n$} & \multirow[b]{2}{*}{ NoneRange } & \multicolumn{6}{|c|}{ Perpetrator-Victims } \\
\hline & & & $\underset{M d n}{\text { Perp-Vic }}$ & $\begin{array}{c}\text { Perp-Vic } \\
\text { Range }\end{array}$ & $\begin{array}{c}\text { Mann-Whitney } \\
U\end{array}$ & $z$-score & $p$-value & $\underset{(r)}{\text { Effect size }}$ \\
\hline Relationship closeness & 4.08 & $1.58-6.25$ & 5.08 & $1.00-7.00$ & 1127.00 & -1.835 & .067 & -0.17 \\
\hline Age & 14.00 & $12.00-18.00$ & 14.00 & $12.00-18.00$ & 1085.00 & -2.842 & $.004^{*}$ & -0.26 \\
\hline
\end{tabular}

Note: *Significant at the .03 level (2-tailed) Bonferroni corrected

Table 3. Multinomial Logistic regression of correlates for TAADVA experience for males (n=105). Reference group is no experience of TAADVA

\begin{tabular}{|c|c|c|c|c|c|c|}
\hline & \multirow[b]{2}{*}{ B $(S E)$} & \multirow[b]{2}{*}{ Wald's $X^{2}$} & \multirow[b]{2}{*}{ P value } & \multicolumn{3}{|c|}{$95 \% \mathrm{CI}$ for $O R$} \\
\hline & & & & Lower & $\operatorname{EXP}(B)$ & Upper \\
\hline \multicolumn{7}{|l|}{ TAADVA Victim Only } \\
\hline Intercept & $18.47(1.96)$ & & & & & \\
\hline Avoidant Attachment & $-0.53(0.28)$ & 3.47 & .063 & 0.34 & 0.59 & 1.03 \\
\hline Controlling ADVA Victim & $0.50(0.92)$ & 0.29 & .591 & 0.27 & 1.64 & 10.04 \\
\hline Physical ADVA Perpetrator & $-16.66(1.15)$ & 209.85 & $.000 * *$ & $6.11 \mathrm{E}-009$ & $5.82 \mathrm{E}-008$ & $5.54 \mathrm{E}-007$ \\
\hline \multicolumn{7}{|l|}{ TAADVA Perpetrator-Victim } \\
\hline Intercept & $22.54(1.48)$ & & & & & \\
\hline Avoidant Attachment & $-0.68(0.28)$ & 6.13 & $.013 *$ & 0.29 & 0.51 & 0.87 \\
\hline Controlling ADVA Victim & $-1.50(0.66)$ & 5.12 & $.024 *$ & 0.06 & 0.22 & 0.82 \\
\hline Physical ADVA Perpetrator & $-18.06(0.00)$ & - & - & $1.43 \mathrm{E}-008$ & $1.43 \mathrm{E}-008$ & $1.43 \mathrm{E}-008$ \\
\hline
\end{tabular}

Note: $R^{2}=.24\left(\right.$ Cox \& Snell), .27 (Nagelkerke). Model $\chi^{2}(6)=28.41, * p<.05 . * * p .001$ 
Table 4. Multinomial Logistic regression of correlates for TAADVA experience for females $(n=149)$. Reference group is no experience of TAADVA

\begin{tabular}{|c|c|c|c|c|c|c|}
\hline & \multirow[b]{2}{*}{$\mathrm{B}(S E)$} & \multirow[b]{2}{*}{ Wald's $X^{2}$} & \multirow[b]{2}{*}{ P value } & \multicolumn{3}{|c|}{ 95\% CI for $O R$} \\
\hline & & & & Lower & $\operatorname{EXP}(B)$ & Upper \\
\hline \multicolumn{7}{|l|}{ TAADVA Victim Only } \\
\hline Intercept & $-15.93(3.97)$ & & & & & \\
\hline Age & $-0.13(0.24)$ & 0.27 & .603 & 0.55 & 0.88 & 1.41 \\
\hline Friend Current TAADVA Victim & $0.11(1.05)$ & 0.01 & .915 & 0.14 & 1.12 & 8.83 \\
\hline $\begin{array}{c}\text { Friend Historical TAADVA } \\
\text { Perpetrator }\end{array}$ & $1.43(1.26)$ & 1.28 & .257 & 0.35 & 4.17 & 49.46 \\
\hline Controlling ADVA Victim & $-2.92(1.08)$ & 7.28 & $.007 * *$ & 0.01 & 0.05 & 0.45 \\
\hline Controlling ADVA Perpetrator & $0.62(0.70)$ & 0.80 & .372 & 0.48 & 1.86 & 7.31 \\
\hline Physical ADVA Victim & $-1.54(1.34)$ & 1.32 & .252 & 0.02 & 0.22 & 2.97 \\
\hline Physical ADVA Perpetrator & $19.81(0.00)$ & - & - & 400150507.7 & 400150507.7 & 400150507.7 \\
\hline \multicolumn{7}{|l|}{ TAADVA Perpetrator-Victim } \\
\hline Intercept & $-0.51(3.00)$ & & & & & \\
\hline Age & $0.30(0.20)$ & 2.20 & .138 & 0.91 & 1.34 & 1.98 \\
\hline Friend Current TAADVA Victim & $-0.81(0.78)$ & 1.08 & .298 & 0.10 & 0.45 & 2.05 \\
\hline $\begin{array}{c}\text { Friend Historical TAADVA } \\
\text { Perpetrator }\end{array}$ & $0.10(0.78)$ & 0.02 & .898 & 0.24 & 1.11 & 5.13 \\
\hline Controlling ADVA Victim & $-3.59(0.97)$ & 13.61 & $.000 * * *$ & 0.00 & 0.03 & 0.19 \\
\hline Controlling ADVA Perpetrator & $-0.31(0.63)$ & 0.24 & .626 & 0.21 & 0.74 & 2.53 \\
\hline Physical ADVA Victim & $-2.33(1.10)$ & 4.45 & $.035^{*}$ & 0.01 & 0.10 & 0.85 \\
\hline Physical ADVA Perpetrator & $2.59(1.14)$ & 5.18 & $.023 *$ & 1.43 & 13.36 & 124.70 \\
\hline
\end{tabular}

Note: $R 2=.44$ (Cox \& Snell), .51 (Nagelkerke). Model $\chi 2(14)=87.54, p<.001 .{ }^{*} p<.05,{ }^{* *} p<.01,{ }^{* * *} p<.001$ 